\title{
Substratos no desenvolvimento de cultivares de gérbera envasada ${ }^{(1)}$
}

\author{
FERNANDA LUDWIG(2); DIRCEU MAXIMINO FERNANDES ${ }^{(3)}$; \\ AMARALINA CELOTO GUERRERO(4) e ROBERTO LYRA VILLAS BÔAS ${ }^{(3)}$
}

\begin{abstract}
RESUMO
A gérbera vem ganhando destaque no mercado brasileiro de flores e plantas ornamentais e com isso há uma crescente demanda quanto à seleção de substratos adequados para sua produção. O presente trabalho foi conduzido com o objetivo de avaliar o crescimento e a distribuição de massa seca e de nitrogênio em plantas de gérbera envasada, em função dos substratos e cultivares. O delineamento experimental utilizado foi o de blocos ao acaso e esquema fatorial $5 \times 2$ ( 5 substratos e 2 cultivares) e 4 repetições. Plantas de gérbera, cultivares Cherry e Red, foram avaliadas aos 1, 15, 29, 43 e 50 dias após aclimatação (DAA) quanto ao número de folhas, à área foliar, à massa seca da parte aérea e ao acúmulo de nitrogênio. A partir dos 29 DAA, a inflorescência estabeleceu-se como dreno e contribuiu significativamente para a massa seca da parte aérea. O acúmulo de nitrogênio ocorreu de forma semelhante nos períodos vegetativo e reprodutivo. O crescimento, a distribuição de massa seca e de nitrogênio em plantas de gérbera de vaso das cultivares Red e Cherry são influenciadas pelas características físicas e químicas dos substratos utilizados, com melhores resultados em substratos com densidade seca inferior a $530 \mathrm{~kg} \mathrm{~m}^{-3}$ e valores de $\mathrm{pH}$ entre 5,5 e 6,8 .
\end{abstract}

Palavras-chave: Gerbera jamesonii, produção, pH, densidade de volume.

\begin{abstract}
Substrates in the development potted gerbera cultivars

The gerbera is a prominent culture that has gained in the Brazilian market of flowers and ornamental plants and there is a great demand for adequate substrates for its production. This study was conducted to evaluate the growth and distribution of dry mass and nitrogen in potted gerbera, depending on the substrates and cultivars. Experimental design was randomized blocks in a $5 \times 2$ factorial arrangement (5 substrates and 2 cultivars). Red and Cherry cultivars were evaluated at 1, 15, 29, 43 and 50 days of acclimation (DAA) to the leaves number, leaf area, dry mass of aerial parts (leaf and flower) and accumulation of nitrogen. From 29 DAA, the inflorescence has established as the drain and has contributed significantly to the total dry mass. The accumulation of nitrogen was similar in vegetative and reproductive periods. The traits evaluated varied depending on the substrate used, influenced by physical and chemical properties of the same. The growth, dry matter and nitrogen distribution potted gerbera cultivars Cherry and Red are influenced by physical and chemical characteristics of substrates, with better results in substrates with bulk density of less than $530 \mathrm{~kg} \mathrm{~m}^{-3}$ and $\mathrm{pH}$ values between 5.5 and 6.8 .
\end{abstract}

Keywords: Gerbera jamesonii, production, $\mathrm{pH}$, bulk density.

\section{INTRODUÇÃO}

A gérbera é uma planta herbácea perene, pertencente à família Asteraceae. É originária da África do Sul e apresenta potencial para ser produzida em várias áreas geográficas, desde que o nível de tecnologia permita (MERCURIO, 2002). Pela importância que a cultura representa no mercado de flores e plantas ornamentais como flor envasada, o estudo das características dos substratos para o seu cultivo é imprescindível (LUDWIG et al., 2011).

O substrato utilizado é um dos fatores tecnológicos que influenciam o desenvolvimento de plantas cultivadas em recipientes, distinguindo-se do cultivo em solo principalmente pela área reduzida para o crescimento das raízes. A limitação do volume exige que o substrato seja capaz de manter a água facilmente disponível à planta sem comprometer a concentração de oxigênio no meio (FERMINO, 2002), apresentar poder de tamponamento para o pH e capacidade de retenção de nutrientes (KAMPF, 2000).

Substratos aerados permitem o desenvolvimento de pelos radiculares finos, o que aumenta a absorção de nutrientes (BELLÉ, 2001). Já a disponibilidade de água atua na fotossíntese, absorção de nutrientes, transporte e síntese de hormônios, entre outros processos fisiológicos. Desse modo, é importante estabelecer uma adequada relação entre ar e água no substrato.

Os valores de $\mathrm{pH}$ e condutividade elétrica (CE) do substrato são critérios químicos que interferem no desenvolvimento das plantas. A importância do pH refere-

\footnotetext{
(1) Trabalho recebido para publicação em 02/03/2013 e aprovado em 09/03/2015

(2) Universidade Estadual do Rio Grande do Sul (UERGS), Unidade em Santa Cruz do Sul. Santa Cruz do Sul-RS, Brasil. * Autor correspondente:

fernanda-ludwig@uergs.edu.br

(3) Universidade Estadual Paulista (UNESP), FCA, Departamento de Recursos Naturais/Ciência do Solo, Botucatu-SP, Brasil.

(4) Universidade Federal de Campina Grande (UFCG), Centro de Ciências e Tecnologia Alimentar, Pombal-PB, Brasil.
} 
se especialmente à disponibilidade de nutrientes, enquanto a CE fornece estimativa do conteúdo de sais solúveis do meio de crescimento. Plantas variam quanto à tolerância ao estresse osmótico causado por altos níveis de salinidade (FERMINO, 1996).

Precocidade, maior número de inflorescências por planta e uniformidade na qualidade comercial são relacionados com a aeração no ambiente radicular e drenagem da água de irrigação (LENZI et al., 1998; MASCARINI, 1998), enquanto a produção também é relacionada com o pH do meio de cultivo (ROGERS e TJIA, 1990).

Dessa forma, o presente trabalho foi conduzido com o objetivo de avaliar o crescimento, a distribuição de massa seca e de nitrogênio em plantas de gérbera envasada, em função dos substratos e cultivares.

\section{MATERIAL E MÉTODOS}

O experimento foi conduzido em casa de vegetação com cobertura plástica transparente de $150 \mu \mathrm{m}$ de espessura, laterais de tela branca, cortinas de plástico transparente e pavimento de concreto, em uma área total de $168 \mathrm{~m}^{2}$ (7 x $24 \mathrm{~m}$ ) e 2,6 m de pé direito. Objetivando conduzir a cultura na faixa máxima de 50.000 lux, o ambiente superior interno da casa de vegetação apresentava uma malha termorefletora $(50 \%)$, manejada manualmente durante o dia. A temperatura média no interior da casa de vegetação foi de $21^{\circ} \mathrm{C}$ e a umidade relativa média do ar foi de $55 \%$.

$\mathrm{O}$ delineamento experimental utilizado foi o de blocos ao acaso e esquema fatorial $5 \times 2$

(5 substratos e 2 cultivares), 4 repetições e 10 plantas por parcela. Os substratos utilizados foram:

- 1: 40\% terra de subsolo (Latossolo Vermelho Escuro), $40 \%$ casca de pinus, $10 \%$ composição $1\left(\mathrm{C}_{1}\right)$, composta por $40 \%$ casca de pinus $+30 \%$ vermiculita $+30 \%$ casca de arroz carbonizada, 10\% composição $2\left(\mathrm{C}_{2}\right)$, composta por $75 \%$ casca de pinus $+25 \%$ acícula de pinus;

- 2: $20 \%$ terra de subsolo, $30 \%$ casca pinus, $30 \% \mathrm{C}_{1}$, $20 \% \mathrm{C}_{2}$;

- 3: $50 \%$ casca de pinus, $20 \%$ fibra de coco granulada, $30 \% \mathrm{C}_{1}$;

- 4: $30 \%$ casca de pinus, $30 \% \mathrm{C}_{1}, 20 \%$ fibra de coco granulada, $20 \%$ fibra de coco mista;

- 5: substrato comercial $(70 \%$ casca de pinus $+15 \%$ turfa $+15 \%$ vermiculita).

Procedeu-se a diluição dos substratos pelo método 1:5 para determinar as características químicas de $\mathrm{CE}$ e $\mathrm{pH}$ (BRASIL, 2007) e o teor dos macronutrientes e micronutrientes (SONNEVELD e ELDEREN, 1994). A relação $\mathrm{C} / \mathrm{N}$ e a matéria orgânica foram quantificadas de acordo com Raij et al. (2001). Determinaram-se também as características físicas de densidade (BRASIL, 2007) e retenção de água (DE BOODT e VERDONCK, 1972). As características dos substratos são apresentadas na Tabela 1.

Foram utilizadas mudas de gérbera (Gerbera jamesonii L.), com quatro folhas definitivas, cultivares Cherry e
Red pertencentes à série Dark eyes da empresa Sakata Seed Sudamérica $\left(\right.$ Sakata $\left.^{\circledR}\right)$, propagadas sexuadamente. As mudas foram transplantadas em vasos com capacidade para $1 \mathrm{~L}$ (11,5 cm de altura, 13,0 cm de diâmetro superior e $9,0 \mathrm{~cm}$ de diâmetro inferior), preenchidos com o substrato correspondente ao tratamento, de acordo com a densidade úmida. Os vasos foram dispostos a cada $25,0 \mathrm{~cm}$, sobre bancadas de madeira.

O manejo da fertirrigação baseou-se na pesagem diária dos vasos, os quais foram mantidos na faixa de 50 a $25 \%$ da água disponível no substrato. A irrigação foi realizada via solução nutritiva com a seguinte composição em $\mathrm{mg} \mathrm{L}^{-1}$ : $121 \mathrm{~N}^{-\mathrm{NO}_{3}^{-}}, 12 \mathrm{~N}_{-} \mathrm{NH}_{4}^{+}, 92 \mathrm{~K}, 24 \mathrm{P}, 175 \mathrm{Ca}, 27 \mathrm{Mg}$ e $39 \mathrm{~S}$, 0,19 B, 0,08 Cu, 2,74 Fe, 0,19 Mn, 0,04 Mo e 0,08 Zn no

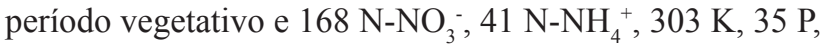
$105 \mathrm{Ca}, 45 \mathrm{Mg}$ e $55 \mathrm{~S}, 0,19 \mathrm{~B}, 0,08 \mathrm{Cu}, 2,74 \mathrm{Fe}, 0,19 \mathrm{Mn}$, $0,04 \mathrm{Mo} \mathrm{e} 0,08 \mathrm{Zn}$ no reprodutivo.

As plantas foram aclimatadas durante 30 dias, quando permaneceram sob intensidade luminosa de 25.000 lx. O número de folhas, a área foliar e a massa seca das plantas foram determinadas aos 1, 15, 29, 43 e 50 dias após aclimatação (DAA). A área foliar foi determinada em medidor modelo Li 3100, da marca Licor. A parte aérea das plantas, separada em folhas e inflorescências no período reprodutivo, iniciado aos 29 DAA, foi seca em estufa de ventilação forçada a $65^{\circ} \mathrm{C}$ por um período de 48 horas, obtendo-se a massa seca em balança digital. Em seguida as amostras foram moídas em moinho tipo "Willey" e determinado o teor de nitrogênio $(\mathrm{N})$ de acordo com Malavolta et al. (1997), obtendo-se o acúmulo a partir do produto da massa seca pela sua concentração.

Os resultados foram submetidos à análise de variância. Os efeitos dos substratos e das cultivares tiveram suas médias comparadas pelo teste Tukey a $5 \%$ de probabilidade, quando significativos. O programa estatístico utilizado foi o Sisvar (FERREIRA, 2011).

\section{RESULTADOS E DISCUSSÃO}

As plantas de gérbera apresentaram número de folhas diferenciado em função dos substratos utilizados, exceto no primeiro dia após aclimatação (Tabela 2), que ocorreu um mês após o transplante das mudas. As plantas conduzidas nos substratos 3 e 4 apresentaram maior número de folhas, sem diferir significativamente daquelas nos substratos 1 e 2 aos 15 DAA e 1 e 5 aos 29 e 50 DAA. De forma geral, as plantas no substrato 2 tiveram menor número de folhas. Aos 43 DAA houve efeito da interação entre cultivares e substratos, sendo o maior número para Cherry no substrato 4.

O número médio de folhas aumentou ao longo do ciclo de crescimento, entretanto, mais acentuado na primeira quinzena após a aclimatação, quando representou $59 \%$ do total. Resultado semelhante foi obtido por Ludwig et al. (2010) em trabalho com cultivares de gérbera de vaso e soluções nutritivas. 
Tabela 1. Características físicas e químicas dos substratos utilizados no crescimento e desenvolvimento de gérbera. Table 1. Physical and chemical characteristics of substrates used in the gerbera growth and development.

\begin{tabular}{|c|c|c|c|c|c|}
\hline \multirow[t]{2}{*}{ Características } & \multicolumn{5}{|c|}{ Substratos } \\
\hline & 1 & 2 & 3 & 4 & 5 \\
\hline \multicolumn{6}{|l|}{ Físicas } \\
\hline Porosidade total- PT (\%) & 78,79 & 80,62 & 84,28 & 89,18 & 83,51 \\
\hline Sólidos (\%) & 21,21 & 19,38 & 15,72 & 10,82 & 16,49 \\
\hline Espaço de aeração - EA (\%) & 23,50 & 19,33 & 12,76 & 17,15 & 21,10 \\
\hline Água disponível - AD (\%) & 28,74 & 29,40 & 37,34 & 35,80 & 32,90 \\
\hline Água remanescente - AR (\%) & 26,48 & 31,89 & 34,62 & 36,23 & 29,50 \\
\hline Densidade úmida $\left(\mathrm{kg} \mathrm{m}^{-3}\right)$ & 750 & 850 & 690 & 590 & 530 \\
\hline Densidade seca $\left(\mathrm{kg} \mathrm{m}^{-3}\right)$ & 538 & 540 & 450 & 298 & 340 \\
\hline \multicolumn{6}{|l|}{ Químicas } \\
\hline $\mathrm{CE}_{1: 5}\left(\mathrm{dS} \mathrm{m}^{-1}\right)$ & 0,54 & 0,49 & 0,26 & 0,28 & 0,48 \\
\hline $\mathrm{pH}_{1: 5 \text { (inicial) }}$ & 5,13 & 4,81 & 5,74 & 5,74 & 7,58 \\
\hline $\mathrm{pH}_{1: 5 \text { (final) }}$ & 5,42 & 4,86 & 5,63 & 5,59 & 6,89 \\
\hline $\mathrm{N}-\mathrm{NO}_{31: 1,5}^{-}\left(\mathrm{mg} \mathrm{L}^{-1}\right)$ & 77,99 & 122,17 & 2,51 & 12,72 & 7,10 \\
\hline $\mathrm{K}_{1: 1,5}\left(\mathrm{mg} \mathrm{L}^{-1}\right)$ & 70,25 & 86,00 & 45,43 & 56,70 & 176,25 \\
\hline $\mathrm{Ca}_{1: 1,5}\left(\mathrm{mg} \mathrm{L}^{-1}\right)$ & 52,40 & 84,98 & 3,57 & 22,83 & 21,63 \\
\hline $\operatorname{Mg}_{1: 1,5}\left(\mathrm{mg} \mathrm{L}^{-1}\right)$ & 50,15 & 57,60 & 12,13 & 24,45 & 12,83 \\
\hline $\mathrm{Cu}_{1: 1,5}\left(\mathrm{mg} \mathrm{L}^{-1}\right)$ & 0,013 & 0,015 & 0,060 & 0,028 & 0,063 \\
\hline $\mathrm{Fe}_{1: 1,5}\left(\mathrm{mg} \mathrm{L}^{-1}\right)$ & 0,383 & 0,62 & 5,568 & 1,763 & 1,825 \\
\hline $\mathrm{Mn}_{1: 1,5}\left(\mathrm{mg} \mathrm{L}^{-1}\right)$ & 0,19 & 2,48 & 0,203 & 0,378 & 0,838 \\
\hline $\mathrm{Zn}_{1: 1,5}\left(\mathrm{mg} \mathrm{L}^{-1}\right)$ & 0,038 & 0,11 & 0,063 & 0,060 & 0,083 \\
\hline
\end{tabular}

Substratos: 1: 40\% terra de subsolo (Latossolo Vermelho Escuro (LVE)), 40\% casca de pinus (CP), 10\% composição 1 (C $\left.\mathrm{C}_{1}\right)(40 \%$ casca de pinus $+30 \%$ vermiculita $+30 \%$ casca de arroz carbonizada), $10 \%$ composição $2\left(\mathrm{C}_{2}\right)(75 \%$ casca de pinus $+25 \%$ acícula de pinus); 2: 20\% LVE, 30\% CP, 30\% $\mathrm{C}_{1}, 20 \% \mathrm{C}_{2} ; 3: 50 \% \mathrm{CP}, 20 \%$ fibra de coco granulada (FCG), $30 \% \mathrm{C}_{1} ; 4: 30 \% \mathrm{CP}, 30 \% \mathrm{C}_{1}, 20 \%$ FCG, 20\% fibra de coco mista; 5 : substrato comercial ( $70 \%$ casca de pinus $+15 \%$ turfa $+15 \%$ vermiculita). CE: condutividade elétrica, PT: porosidade total, S: sólidos, EA: espaço de aeração, AD: água disponível, AR: água remanescente, DU: densidade úmida, DS: densidade seca, DP: distribuição de partículas.CE: eletrical condutivity, PT: total porosity, S: solids, EA: aeration space, AD: available water, AR: remaining water, DU: humid bulk density, DS: dry bulk density, DP: particles distribution.

As plantas apresentaram área foliar diferenciada em função dos substratos (Tabela 3). Diferença entre cultivares foi registrada aos 15,43 e 50 DAA, maior para Cherry e o efeito da interação entre substratos e cultivares, aos 15 e 43 DAA. No período vegetativo, a média geral para essa característica foi superior nos substratos 3 e 4 , e sem diferir significativamente no substrato 5 aos 50 DAA. De forma geral, as plantas desenvolveram maior área foliar quando conduzidas no substrato 4 e menor no substrato 2 .

A área foliar foi crescente ao longo do ciclo, com $42 \%$ do total aos 15 DAA, concordando com o número de folhas. Os menores incrementos ocorreram dos 29 aos 43 DAA, quando inicia a emissão das inflorescências e há uma tendência de deslocamento dos assimilados para a formação destas. De acordo com Larcher (1986) as plantas de ciclo curto apresentam a tendência de usar uma maior proporção de fotoassimilados para a formação de folhas no início do crescimento, gerando grande quantidade de tecido em reduzido período de tempo.
O desenvolvimento da área foliar nas plantas conduzidas no substrato 5 foi lento durante o período de aclimatação, semelhante àquelas produzidas no substrato 2 (Tabela 3). Do início do período avaliativo até aos 15 DAA, o incremento na área foliar foi somente de $165 \mathrm{~cm}^{2}$, passando para $389 \mathrm{~cm}^{2}$ dos 15 aos 29 DAA. Esse rápido desenvolvimento da área foliar foi importante para que as plantas no substrato 5 se assemelhassem de forma significativa daquelas plantas já inicialmente produzidas nos substratos 3 e 4 .

A limitação que o substrato 5 impôs ao crescimento inicial da cultura deve-se ao seu valor de $\mathrm{pH}$ elevado $(7,58)$, uma vez que as características físicas eram adequadas (Tabela 1). O impulso no crescimento ao longo do ciclo relaciona-se com a redução do $\mathrm{pH}$, com valores finais de 6,89 , já que as demais características permaneceram constantes entre os substratos. De acordo com Rogers e Tjia (1990), o pH para gérbera pode ser mantido entre 5,5 e 6,5 . 
Tabela 2. Número de folhas em plantas de gérbera de vaso, em função dos substratos e cultivares.

Table 2. Leaf number in potted gerbera, depending on the substrates and cultivars.

\begin{tabular}{|c|l|l|l|l|l|l|}
\hline \multicolumn{7}{|c|}{ Número de folhas } \\
\hline \\
\hline
\end{tabular}

Substratos: 1: 40\% terra de subsolo (Latossolo Vermelho Escuro (LVE)), 40\% casca de pinus (CP), 10\% composição 1 (C $\left.\mathrm{C}_{1}\right)(40 \%$ casca de pinus $+30 \%$ vermiculita $+30 \%$ casca de arroz carbonizada), $10 \%$ composição $2\left(\mathrm{C}_{2}\right)(75 \%$ casca de pinus $+25 \%$ acícula de pinus $)$; 2: $20 \%$ LVE, $30 \%$ CP, $30 \% \mathrm{C}_{1}, 20 \% \mathrm{C}_{2} ; 3: 50 \% \mathrm{CP}, 20 \%$ fibra de coco granulada (FCG), 30\% C $;$ 4:30\% CP, 30\% C $, 20 \% \mathrm{FCG}, 20 \%$ fibra de coco mista; 5 : substrato comercial ( $70 \%$ casca de pinus $+15 \%$ turfa $+15 \%$ vermiculita). Médias seguidas pela mesma letra não diferem entre si pelo teste Tukey a $5 \%$, minúscula na coluna e maiúscula na linha. S: substrato. C: cultivar. NS: não significativo; *,** significativo a 1 e 5\%, respectivamente. Means followed by the same letters did not differ by the Tukey test at 5\%, lowercase on column and uppercase on line. C: cultivar, NS: not significant; ** significant at 1\%, * significant at 5\%.

Tabela 3. Área foliar em plantas de gérbera de vaso, em função dos substratos e cultivares.

Table 3. Leaf area in potted gerbera, depending on the substrates and cultivars.

\begin{tabular}{|c|c|c|c|c|c|c|c|}
\hline & \multicolumn{7}{|c|}{ Área Foliar } \\
\hline & \multicolumn{7}{|c|}{ DAA } \\
\hline & 1 & \multicolumn{2}{|c|}{15} & 29 & \multicolumn{2}{|c|}{43} & 50 \\
\hline & & \multicolumn{2}{|c|}{ cultivar } & & \multicolumn{2}{|c|}{ cultivar } & \\
\hline & & Red & Cherry & & Red & Cherry & \\
\hline Substrato & \multicolumn{7}{|c|}{ - } \\
\hline 1 & $186 \mathrm{ab}$ & $314 \mathrm{~b} \mathrm{~A}$ & $383 \mathrm{~b} \mathrm{~A}$ & $610 \mathrm{~b}$ & $715 \mathrm{~b} \mathrm{~A}$ & $726 \mathrm{~cd} \mathrm{~A}$ & $889 \mathrm{~b}$ \\
\hline 2 & $146 \mathrm{~b}$ & $243 \mathrm{~b} \mathrm{~B}$ & $340 \mathrm{~b} \mathrm{~A}$ & $424 \mathrm{c}$ & 487 c A & $594 \mathrm{~d} \mathrm{~A}$ & $664 \mathrm{c}$ \\
\hline 3 & $204 \mathrm{a}$ & 463 a $A$ & $362 \mathrm{~b} \mathrm{~B}$ & $749 a b$ & $877 \mathrm{ab} A$ & 924 bc A & $1226 a$ \\
\hline 4 & $219 a$ & 441 a B & 533 a A & $856 \mathrm{a}$ & 1064 a B & 1393 a A & $1287 \mathrm{a}$ \\
\hline 5 & $139 \mathrm{~b}$ & $287 \mathrm{~b} \mathrm{~A}$ & $323 \mathrm{~b} \mathrm{~A}$ & $693 \mathrm{~b}$ & 948 a A & 997 b A & $1186 \mathrm{a}$ \\
\hline \multicolumn{8}{|l|}{ Cultivar } \\
\hline Red & 167 & & & 634 & & & 994 B \\
\hline Cherry & 190 & & & 699 & & & $1108 \mathrm{~A}$ \\
\hline $\mathrm{S}$ & $* *$ & \multicolumn{2}{|c|}{$* *$} & $* *$ & \multicolumn{2}{|c|}{$* *$} & $* *$ \\
\hline $\mathrm{C}$ & NS & \multicolumn{2}{|c|}{$*$} & NS & \multicolumn{2}{|c|}{$* *$} & $*$ \\
\hline $\mathrm{S} * \mathrm{C}$ & NS & \multicolumn{2}{|c|}{$* *$} & NS & \multicolumn{2}{|c|}{$*$} & NS \\
\hline CV (\%) & 21,06 & \multicolumn{2}{|c|}{14,68} & 15,97 & \multicolumn{2}{|c|}{12,27} & 13,07 \\
\hline
\end{tabular}

Substratos: 1: 40\% terra de subsolo (Latossolo Vermelho Escuro (LVE)), 40\% casca de pinus (CP), 10\% composição 1 ( $\left.\mathrm{C}_{1}\right)(40 \%$ casca de pinus $+30 \%$ vermiculita $+30 \%$ casca de arroz carbonizada), $10 \%$ composição $2\left(\mathrm{C}_{2}\right)(75 \%$ casca de pinus $+25 \%$ acícula de pinus $)$; 2: $20 \%$ LVE, 30\% CP, 30\% $\mathrm{C}_{1}, 20 \% \mathrm{C}_{2} ; 3: 50 \% \mathrm{CP}, 20 \%$ fibra de coco granulada (FCG), 30\% C $;$ 4:30\% CP, 30\% C $, 20 \% \mathrm{FCG}, 20 \%$ fibra de coco mista; 5 : substrato comercial ( $70 \%$ casca de pinus $+15 \%$ turfa $+15 \%$ vermiculita). Médias seguidas pela mesma letra não diferem entre si pelo teste Tukey a $5 \%$, minúscula na coluna e maiúscula na linha. S: substrato. C: cultivar. NS: não significativo; *,** significativo a 1 e 5\%, respectivamente. Means followed by the same letters did not differ by the Tukey test at $5 \%$, lowercase on column and uppercase on line. C: cultivar, NS: not significant; ** significant at 1\%, * significant at $5 \%$. 
Valores de $\mathrm{pH}$ reduzidos também influenciam no desenvolvimento da gérbera. As menores áreas foliares obtidas no substrato 2 durante todo o ciclo, tem relação com o reduzido $\mathrm{pH}$, inferior a 5,0 (Tabela 1). Valores de $\mathrm{pH}$ abaixo de 5,8 podem aumentar a disponibilidade de micronutrientes, como o $\mathrm{Fe}$ e o $\mathrm{Mn}$ e ocasionar toxidade (CAVINS et al., 2000; FERMINO, 1996). A elevada densidade do substrato 2 (Tabela 1) também pode ter interferido nos resultados, pois reduz o volume de poros ocupado por ar (BUNT, 1984), limitando o crescimento das plantas.

A área foliar é uma característica inerente à cultivar, superior para Cherry ao final do ciclo. Diferenças entre cultivares para área foliar de gérbera de vaso foram registradas também por Ludwig et al. (2010), ao avaliar as cultivares Cherry, Golden Yellow, Salmon Rose e Orange.

Tabela 4. Massa seca em plantas de gérbera de vaso, em função dos substratos e cultivares.

Table 4. Dry matter in potted gerbera, depending on the substrates and cultivars.

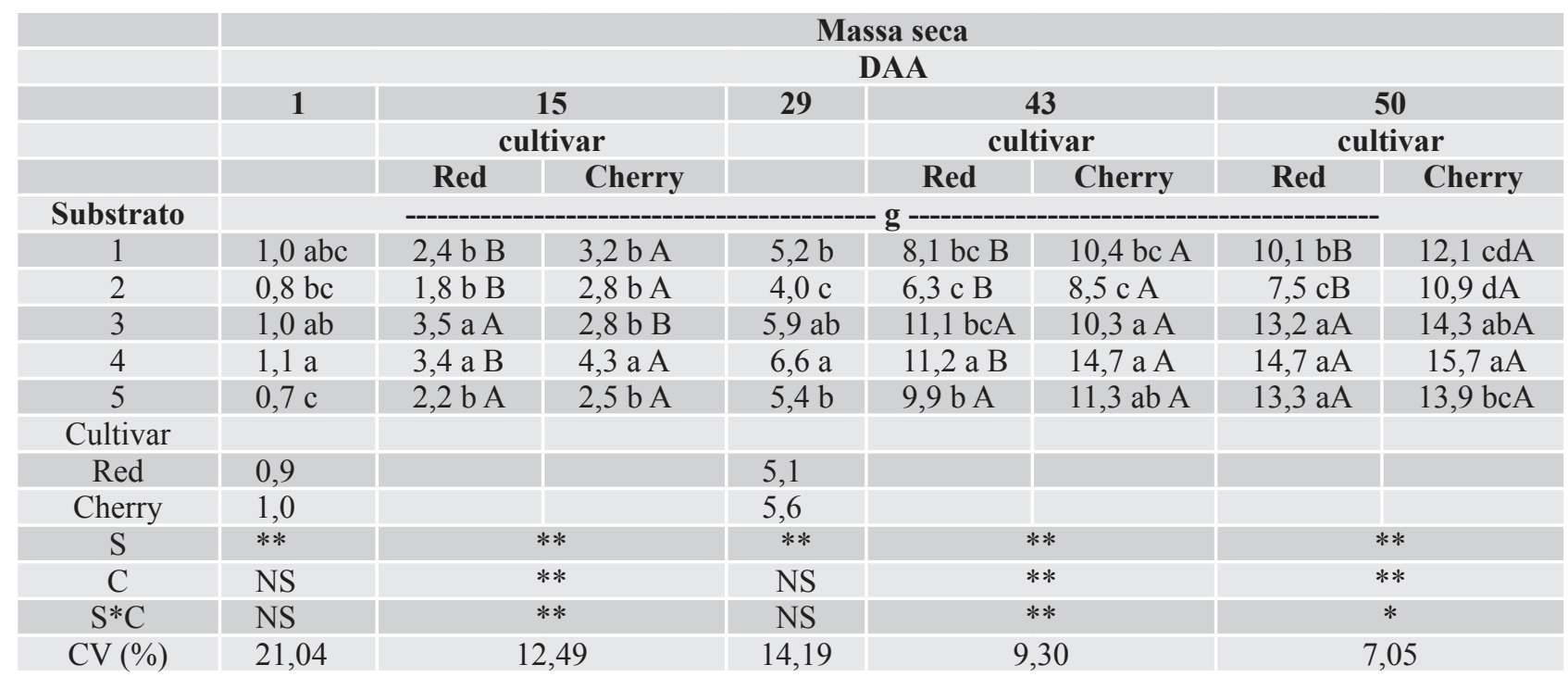

Substratos: 1: 40\% terra de subsolo (Latossolo Vermelho Escuro (LVE)), 40\% casca de pinus (CP), 10\% composição 1 (C $\left.\mathrm{C}_{1}\right)(40 \%$ casca de pinus $+30 \%$ vermiculita $+30 \%$ casca de arroz carbonizada), $10 \%$ composição $2\left(\mathrm{C}_{2}\right)(75 \%$ casca de pinus $+25 \%$ acícula de pinus); 2: $20 \%$ LVE, $30 \%$ CP, 30\% C, $20 \% \mathrm{C}_{2}$; 3: 50\% CP, 20\% fibra de coco granulada (FCG), 30\% C, 4:30\% CP, 30\% C , 20\% FCG, 20\% fibra de coco mista; 5 : substrato comercial ( $70 \%$ casca de pinus $+15 \%$ turfa $+15 \%$ vermiculita). Médias seguidas pela mesma letra não diferem entre si pelo teste Tukey a 5\%, minúscula na coluna e maiúscula na linha. S: substrato. C: cultivar. NS: não significativo; ${ }^{*}, * *$ significativo a 1 e $5 \%$, respectivamente. Means followed by the same letters did not differ by the Tukey test at $5 \%$, lowercase on column and uppercase on line. C: cultivar, NS: not significant; ** significant at 1\%, * significant at $5 \%$.

Os menores valores de massa seca da parte aérea foram observados nas plantas conduzidas no substrato 5 , sem diferir significativamente no 1 e 2 ao 1 DAA e no substrato 2 aos 29 DAA (Tabela 4). A interação entre substratos e cultivares foi verificada aos 15, 43 e 50 DAA. A cultivar Cherry apresentou maior massa seca quando as plantas foram conduzidas nos substratos 1, 2 e 4 aos 15 e 43 DAA, 1 e 2 aos 50 DAA e 1 , 2 e 4 aos 15 DAA. A menor massa seca obtida nas plantas em substratos com valores de $\mathrm{pH}$ acima de 7,0 no substrato 5 e abaixo de 5,0 no substrato 2 (Tabela 1) é consistente com resultados obtidos por Caballero et al. (2009).

A massa seca apresentou maiores acréscimos dos 29 aos 43 DAA, coincidindo com a emissão das inflorescências. Aos 29 DAA, a porcentagem de massa seca correspondente à parte vegetativa era de $88 \%$, reduzindo para $58 \%$ aos 43 e 50 DAA, quando a parte reprodutiva passou a representar $42 \%$ da massa total.

Conforme padrão nas produções comerciais, até os
29 DAA ocorreu a remoção das inflorescências precoces em desenvolvimento, resultando no contínuo acúmulo de massa seca nos órgãos vegetativos. A partir deste período, as inflorescências foram mantidas, estabelecendo-se como drenos, pois grande parte da massa seca total é deslocada para seu estabelecimento (Figura 1).

A distribuição de massa seca entre os diferentes órgãos de uma planta é o resultado final de um conjunto de processos metabólicos e de transporte, que governam o fluxo de assimilados através de um sistema fonte-dreno (DUARTE e PEIL, 2010). Apesar da grande contribuição das inflorescências na massa seca total, as folhas continuaram a se desenvolver e contribuir para a massa total. Essa característica tem importância fundamental para plantas envasadas, em que a qualidade está relacionada com o conjunto de inflorescências e folhas, formando um conjunto equilibrado entre a parte vegetativa e reprodutiva (LUDWIG et al., 2010). 

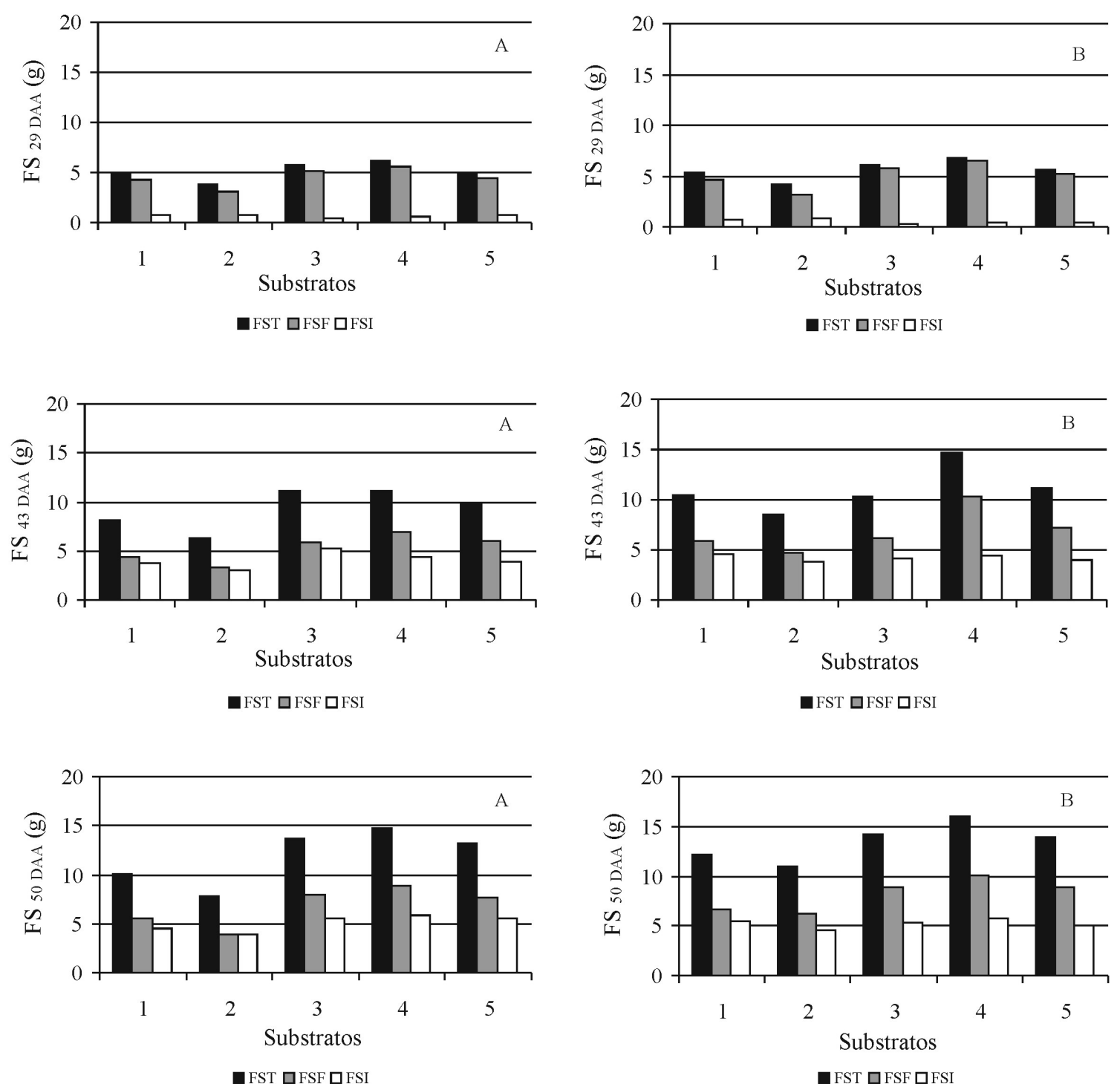

Figura 1. Distribuição da massa seca total (FST) em massa seca das folhas (FSF) e massa seca das inflorescências (FSI) para as cultivares Red (A) e Cherry (B) aos 29, 43 e 50 DAA.

Figure 1. Total dry matter distribution (FST) in leaves dry matter (FSF) and inflorescences dry matter (FSI) for the cultivars Red (A) and Cherry (B) at 29, 43 and 50 DAA.

A maior proporcionalidade para o acúmulo de $\mathrm{N}$ nas inflorescências e folhas ocorreu para as plantas conduzidas nos substratos 1, 2 e 3 aos 43 DAA e 1 e 2 aos 50 DAA (Figura 2). As plantas nos substratos 3, 4 e 5 apresentaram maior acúmulo de massa seca nas folhas em relação aos substratos 1 e 2, o que possivelmente tenha acarretado um maior acúmulo de $\mathrm{N}$, já que este nutriente é responsável principalmente pelo crescimento vegetativo. $\mathrm{O}$ acúmulo de $\mathrm{N}$ nas folhas aos 43 DAA correspondia a $59 \%$ do total, atingindo $64 \%$ aos 50 DAA, indicando sua importância no desenvolvimento da parte vegetativa.

A cultivar Red apresentou menor incremento no acúmulo de $\mathrm{N}$ dos 43 aos 50 DAA quando conduzida no substrato 2, com $21 \mathrm{mg}$ planta ${ }^{-1}$. O maior incremento ocorreu quando conduzida no substrato 5, com $98 \mathrm{mg} \mathrm{planta}^{-1}$. A cultivar Cherry apresentou redução de incremento dos 43 aos 50 DAA quando produzida no substrato 4, de $13 \mathrm{mg}$ planta $^{-1}$ na folha, sendo o acúmulo total devido somente a inflorescência. $O$ maior incremento ocorreu quando conduzida no substrato 3, com $137 \mathrm{mg}$ planta $^{-1}$.

A remobilização de $\mathrm{N}$ das folhas para as inflorescências não atendeu um mesmo padrão e foi dependente do substrato e cultivar. As menores discrepâncias entre inflorescência e folha ocorreram nos substratos 1 e 2 para Red. De forma geral, a porcentagem de $\mathrm{N}$ para as inflorescências em ponto de comercialização foi de $42 \%$ para Red e $36 \%$ para Cherry. De acordo com Marschner (1995), o N translocase facilmente para as partes em crescimento vegetal, sendo mobilizado das folhas maduras e retranslocado para órgãos novos em crescimento. 

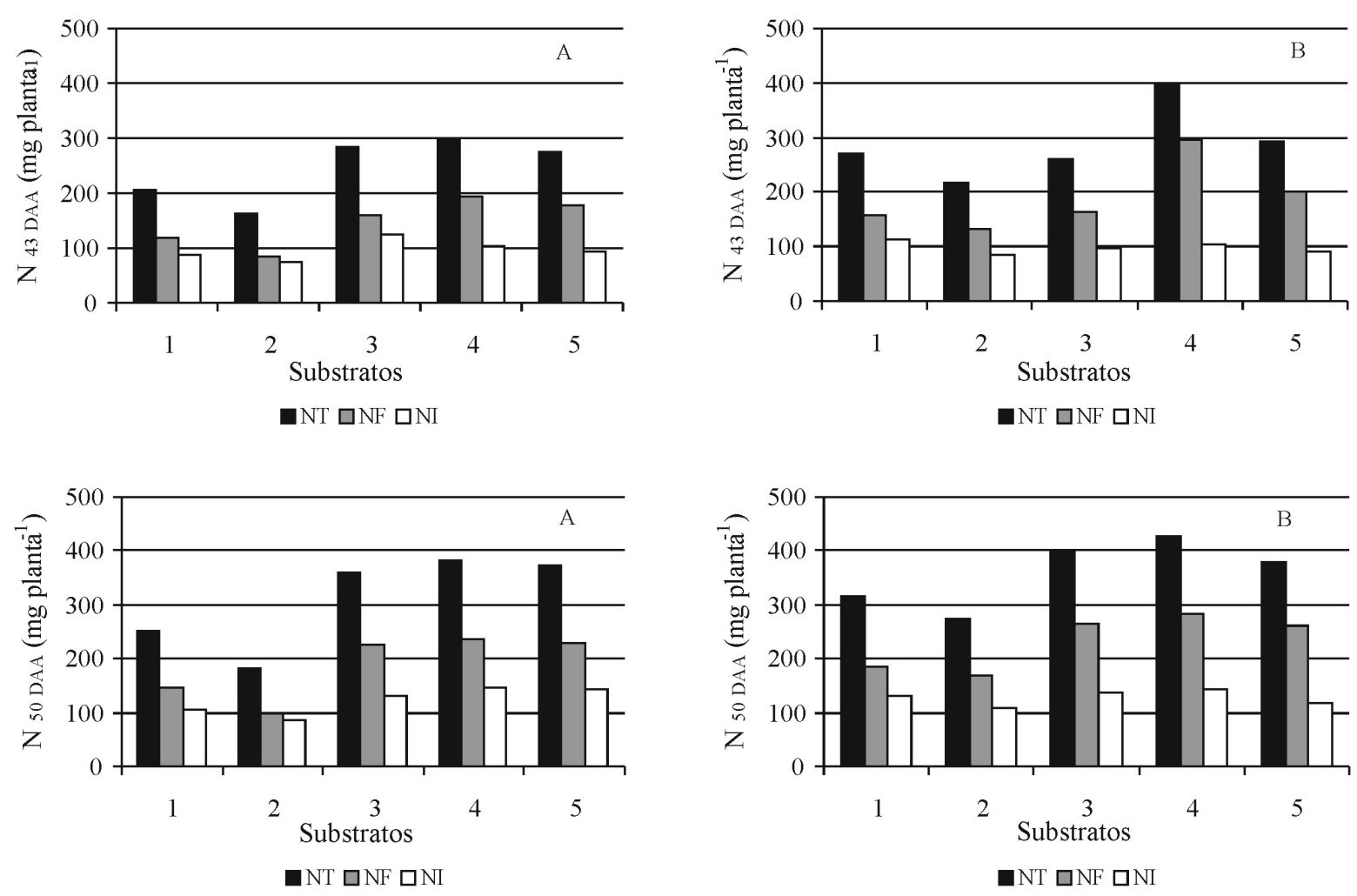

Figura 2. Distribuição do acúmulo de $\mathrm{N}\left(\mathrm{mg} \mathrm{planta}^{-1}\right)$ em $\mathrm{N}$ total $(\mathrm{NT}), \mathrm{N}$ nas folhas $(\mathrm{NF})$ e $\mathrm{N}$ nas inflorescências (NI) para as cultivares Red (A) e Cherry (B) aos 43 e 50 DAA.

Figure 2. $N$ accumulation distribution $\left(\right.$ mg plant $\left.^{-1}\right)$ in total $N(N T)$, leaf $N(N F)$ and $N$ inflorescences (NI) for the cultivars Red (A) and Cherry (B) at 29, 43 and 50 DAA.

Os dados indicam que a demanda de $\mathrm{N}$ ocorre de forma semelhante nos períodos vegetativo e reprodutivo, o que possivelmente relaciona-se com a contínua produção de massa seca nas folhas, parte vegetativa e maior acumuladora desse nutriente. A disponibilidade de $\mathrm{N}$ na solução nutritiva aplicada diariamente, acompanhada do crescente acúmulo de $\mathrm{N}$ no tecido, indica requerimento contínuo desse nutriente, conforme observado também por Ludwig et al. (2008) para diferentes cultivares de gérberas de vaso. Sua característica de perenidade (MERCURIO, 2002) justifica essa característica.

\section{CONCLUSÃO}

O crescimento, a distribuição de massa seca e de nitrogênio em plantas de gérbera de vaso das cultivares Red e Cherry são influenciadas pelas características físicas e químicas dos substratos utilizados, com melhores resultados em substratos com densidade seca inferior a 530 $\mathrm{kg} \mathrm{m}^{-3} \mathrm{e}$ valores de $\mathrm{pH}$ entre 5,5 e 6,8 .

\section{REFERÊNCIAS}

BELLÉ, S. Escolha do substrato. In: KÄMPF, A.N. (coord). Manutenção de plantas ornamentais para interiores, Rígel. 2 edição. 2001. 112p.
BRASIL. Instrução Normativa n.17, de 21 de maio de 2007. Aprova os Métodos Analíticos Oficiais para Análise de Substratos e Condicionadores de Solos. Diário Oficial da União, Brasília, 24 de maio de 2007. Seção 1, p.8.

BUNT, A.C. Physical properties of mixtures of peats and minerals of different particle size and bulk density for potting substrates. Acta Horticulturae, Wageningen, v.150, p.143-153, 1984.

CABALLERO, R.; PAJUELO, P.; ORDOVÁS, J.; CARMONA, E.; DELGADO, A. Evaluation and correction of nutrient availability to Gerbera jamesonii $\mathrm{H}$. Bolus in various compost-based growing media. Scientia Horticulturae, Amsterdam, v.122, p.244-250, 2009.

CAVINS, T.J.; WHIPKER, B.E.; FONTENO, W.C.; HARDEN, B.; McCALL, I.; GIBSON, J.L. Monitoring and managing $\mathrm{pH}$ and $\mathrm{EC}$ using the PourThru extraction method. Raleigh: Horticulture Information, 2000. 17 p.

De BOODT, M.; VERDONCK, O. The physical properties of the substrates in horticulture. Acta Horticulturae, Wageningen, v.26, p.37-44, 1972. 
DUARTE, T.S.; PEIL, R.M.N. Relações fonte: dreno e crescimento vegetativo do meloeiro. Horticultura Brasileira, Brasília, v.28, p.271-276, 2010.

FERMINO, M. H. Aproveitamento de Resíduos Industriais e Agrícolas como alternativas de Substratos Hortícolas. 1996. 91f. Dissertação (Mestrado em Fitotecnia) - Faculdade de Agronomia, Universidade Federal do Rio Grande do Sul, Porto Alegre.

FERMINO, M.H.O uso da análise física na avaliação da qualidade de componentes e substratos. In: FURLANI, A.M.C. et al. Caracterização, manejo e qualidade de substratos para a produção de plantas. Campinas: Instituto Agronômico, 2002. p.29-37.

FERREIRA, D.F. Sisvar: a computer statistical analysis system. Ciência e Agrotecnologia, Lavras, v.35, n.6, p.1039-1042, 2011.

KÄMPF, A. N. Produção comercial de plantas ornamentais. Guaíba, 2000. 254p.

LARCHER, W. Ecofisiologia vegetal. São Paulo: EPU, 1986. 319p.

LENZI, A.; RINALDI, M.; BIOCCA, M.; MALETTA. $\mathrm{M}$; PERGOLA, G. Soilless cultivation of Gerbera in various substrates and two fertirrigation regimes [Gerbera jamesonii Bolus]. Colture-Protette, v. 27, p. 34-38, 1998.

LUDWIG, F.; FERNANDES, D.M.; MOTA, P.R.D.; VILLAS BOAS, R.L. Macronutrientes em cultivares de gérbera sob dois níveis de fertirrigação. Horticultura Brasileira, Brasília, v.26, p.68-73, 2008.
LUDWIG, F.; GUERRERO, A.C.; FERNANDES, D.M.; VILLAS BOAS, R.L. LASCHI, D. Qualidade de cultivares de gérbera de vaso em função das características físicas e químicas dos substratos. Revista Brasileira de Horticultura Ornamental, Campinas, v.17, p.133-140. 2011.

LUDWIG, F.; FERNANDES, D.M; MOTA, P.R.D.; VILLAS BOAS, R.L. Crescimento e produção de gérbera fertirrigada com solução nutritiva. Horticultura Brasileira, Brasília, v.28, p.424-429, 2010.

MALAVOLTA, E.; VITTI, G.C.; OLIVEIRA, S.A. Avaliação do estado nutricional das plantas: princípios e aplicações. Piracicaba: Potafos. 1997. 319p.

MARSCHNER, H. Mineral nutrition of higher plants. San Diego: Academic Press, 1995. 889p.

MASCARINI, L. Gerbera cultivation in growing media. Horticultura Internacional, Tarragona, v.6, p.86-88, 1998.

MERCURIO, G. Gerbera cultivation in greenhouse. The Netherlands: Schreurs, 2002. 206p.

RAIJ, B.V.; ANDRADE, J.C.; CANTARELLA, H.; QUAGGIO, J.A. Análise química da fertilidade dos solos tropicais. Campinas: IAC, 2001. 285p.

ROGERS, M.N.; TJIA, B.O. Gerbera production. Timber Press Growers handbook series, v.4, 1990. 116p.

SONNEVELD, C.; ELDEREN, C.W. Chemical analysis of peaty growing media by means of water extraction. Communication on Soil Science and Plant Analysis, New York, v.25, p.3199-3208, 1994. 